\title{
Contribution à l'étude des Trématodes d'Amphibiens du Cameroun
}

\author{
par Michelle GASSMANN \\ Institut de zoologie ( $\mathrm{P}^{r}$ A. Aeschliman), \\ Université de Neuchâtell, Suisse.
}

\section{Résumé.}

Quelques 800 Amphibiens Anoures appartenant à 88 espèces différentes ont été disséqués en vue de la recherche de parasites intestinaux, pulmonaires et vésicaux. 37 espèces d'Anoures (244 individus) hébergeaient des Trématodes. Nous avons trouvé neuf espèces déjà signalées en Afrique. Une nouvelle espèce est décrite, Haematoloechus ocellati n. sp, parasite pulmonaire d'Hyperolius ocellatus purpurescens. Nous proposons le transfert de l'espèce Haplometroides eburnense Maeder, 1969 dans le genre Plagitura Holl, 1928. De nombreuses espèces sont examinées pour la première fois dans le cadre d'une recherche helminthologique. Ce travail apporte de nouveaux éléments à la biogéographie des Trématodes d'Amphibiens africains et élargit l'éventail des hôtes pour les espèces déjà signalées.

\section{Summary.}

Contribution to the study of Trematodes in Amphibians from the Cameroon.

Some 800 Amphibians representing 88 species were dissected for parasitic Trematodes of the digestive tract, the lungs and the bladder. 37 species of Anurans (244 specimens) harboured Trematodes. Nine species already found in Africa were observed. A new species, Haematoloechus ocellati n. sp. parasite of the lungs, is described. We propose the transfer of the species Haplometroides eburnense Maeder, 1969 into the genus Plagitura Holl, 1928. A large number of Anurans were examined for the first time in order to study Helminthes. This work brings in new elements in the biogeography of african Trematodes Amphibians and increases the number of hosts for already mentioned species.

Ce travail a bénéficié d'un subside du Fonds national de la Recherche scientifique, requête $\mathrm{N}^{\circ} 3.651 .71$. 


\section{Introduction}

Au cours de deux missions au Cameroun (octobre à décembre 1972 et mars à mai 1973), nous avons disséqué environ 800 Amphibiens Anoures en vue d'une étude épidémiologique. La détermination des hôtes a été faite généralement sur le terrain, peu après les captures, par les $\mathrm{D}^{r s}$ J.-L. Perret (Genève) et J.-L. Amiet (Yaoundé), spécialistes reconnus des Amphibiens africains. Nous les remercions ici vivement de leur précieuse collaboration. La liste des animaux examinés (tableau $I$ ) compte 88 espèces dont plusieurs sont endémiques au Cameroun ou examinées pour la première fois dans le cadre de recherches parasitologiques. Nous avons prospecté :

- les environs de Yaoundé, dans un rayon de $100 \mathrm{~km}$, c'est-à-dire un hautplateau de 800-1 $500 \mathrm{~m}$ d'altitude, recouvert par la forêt équatoriale, mais où se trouvent aussi des îlots de savane ;

- la région montagneuse de l'Ouest appelée «Dorsale camerounaise », qui fait frontière avec le Nigeria- et ou de nombreuses espèces d'Amphibiens sont endémiques ;

- le Sud-Ouest, région forestière qui touche au Gabon (fig. 1).

Dans le présent travail, nous n'étudions que les Trématodes, qui appartiennent aux espèces suivantes : Haematoloechus darcheni Combes et Knoepffler, 1967 ; H. micrurus Rees, 1964 ; Metahaematoloechus exoterorchis (Rees, 1964) Yamaguti, 1971; H. ocellati n. sp., parasites pulmonaires; Ostioloides rappiae (Szidat, 1932) Odening, 1960 ; Mesocoelium monas Freitas, 1958; M. gabonensis Maeder, Combes et Knoepffler, 1969 ; Haplometroides eburnense Maeder, 1969, parasites intestinaux ; Diplodiscus subclavatus (Goeze, 1782) et Progonimodiscus doyeri (Ortlepp, 1926), parasites du rectum.

Les autres parasites (Nématodes, Cestodes et Monogènes Polystomatidae) feront l'objet d'une note séparée.

\section{Haematoloechus darcheni}

Combes et Knoepffler, 1967

HôTE : Conraua crassipes.

HaBitat : poumons.

Localites: Kala, Sakbayémé, Ebamina.

FRÉQUENCE D'INFESTATION : 3 individus parasités sur 10 disséqués.

Matériel Étudié : 2 Trématodes adultes et 3 juvéniles colorés et montés in toto. Seuls les adultes ont été mesurés.

IONGUEUR : $3,5-4,3 \mathrm{~mm}$.

LARGEUR: $0,97-1,1 \mathrm{~mm}$.

Ventouse BucCALE: 237-355 $\mu \mathrm{m}$. 


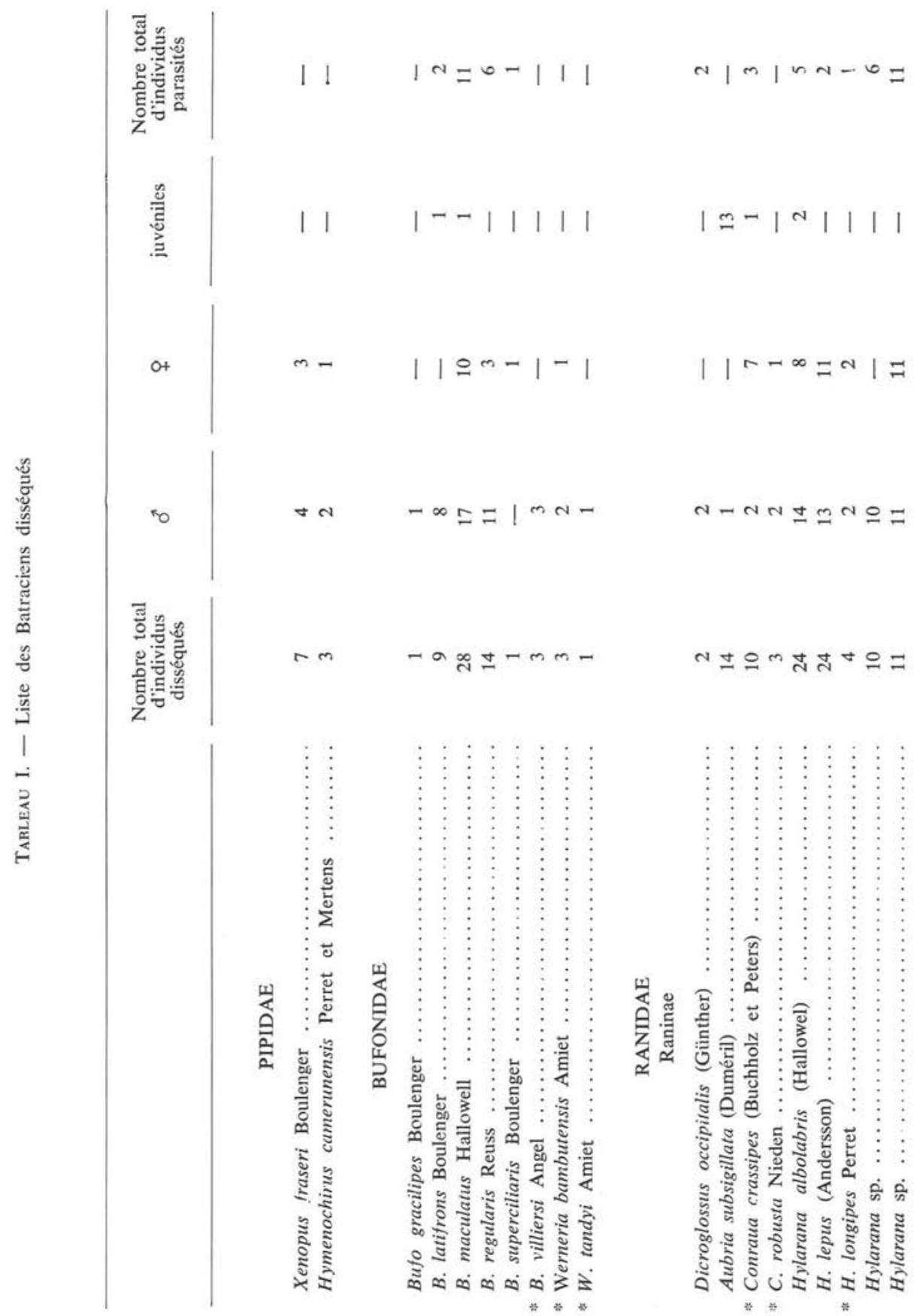




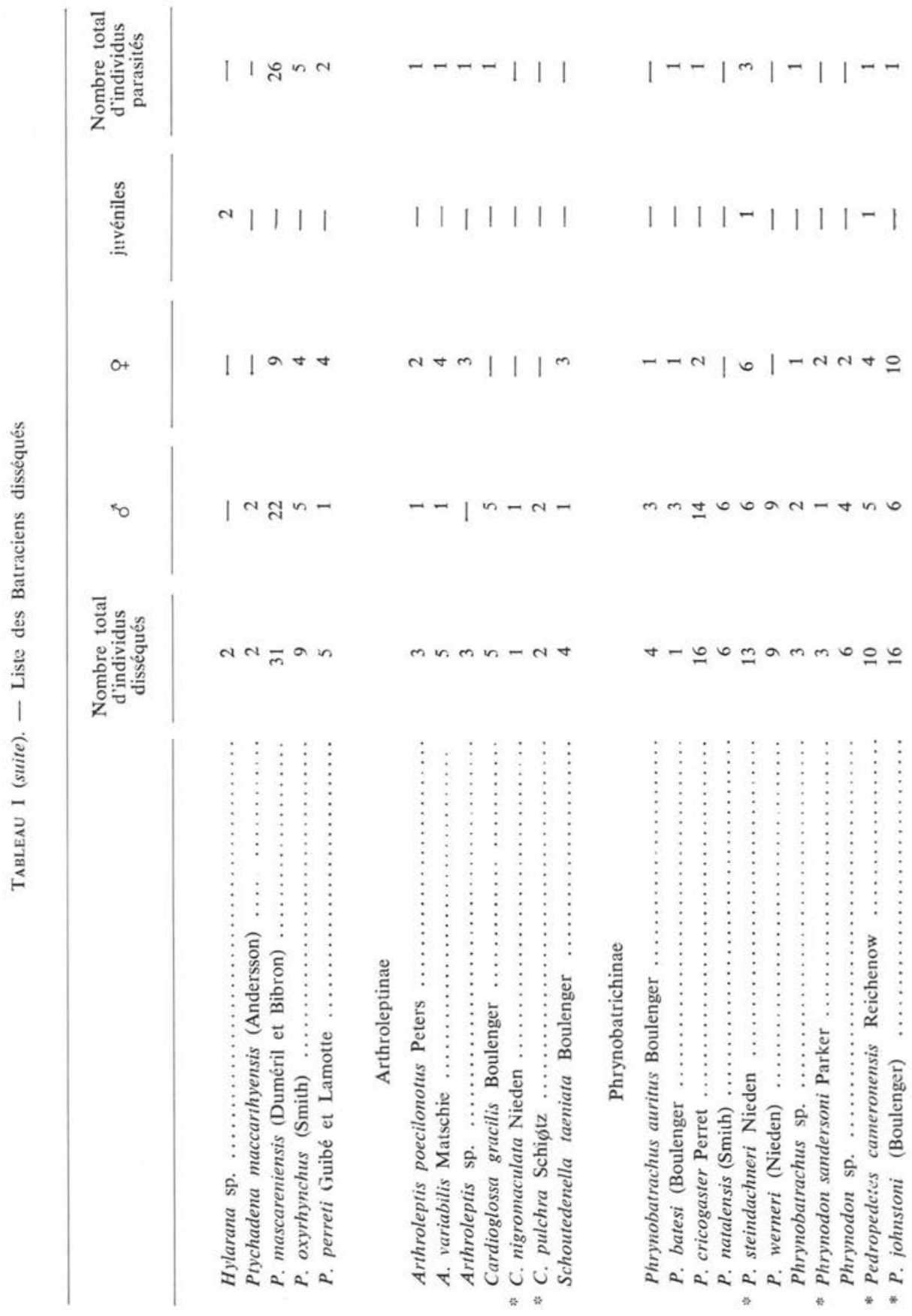


TREMATODES D'AMPHIBIENS DU CAMEROUN

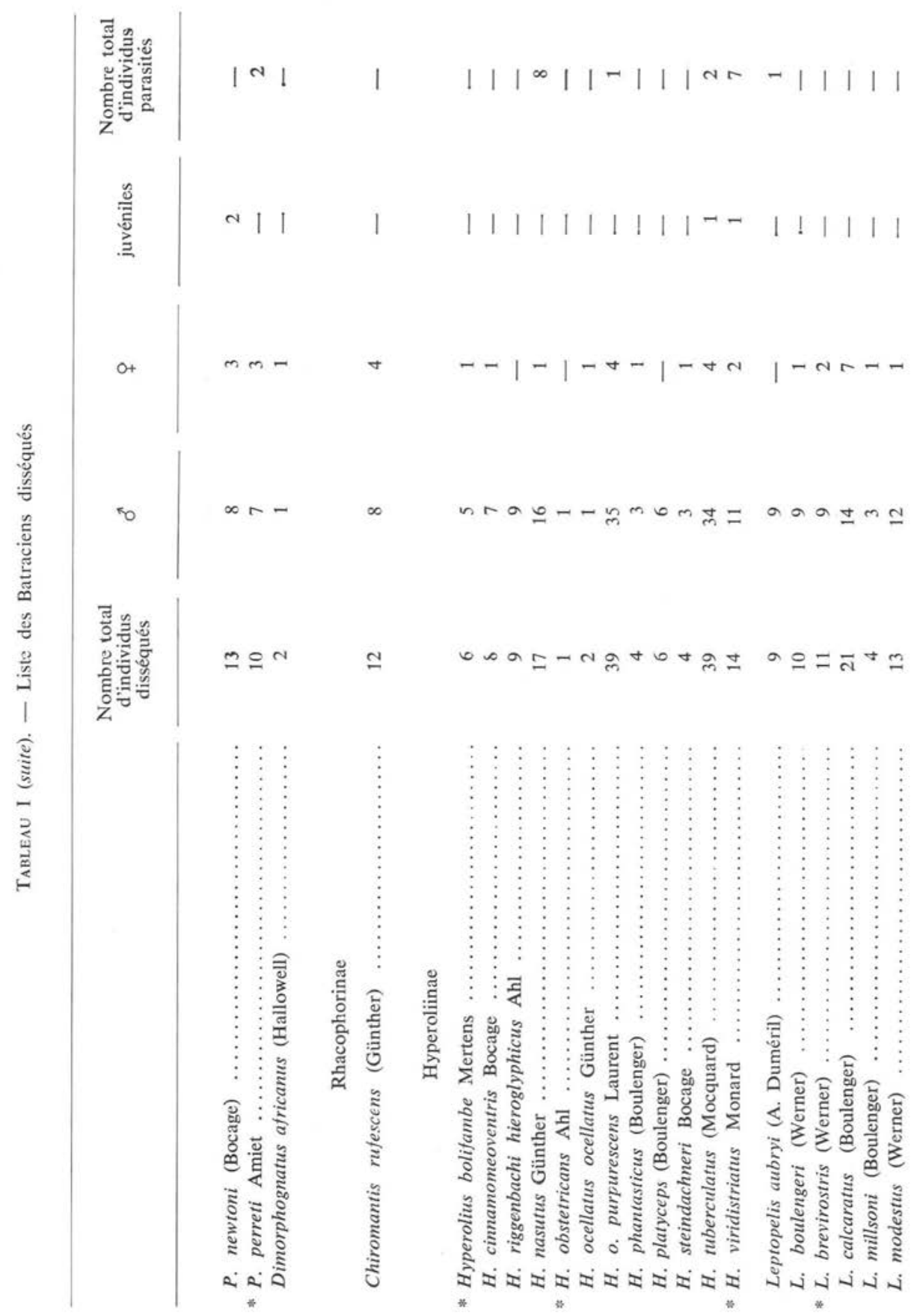


MICHELLE GASSMANN

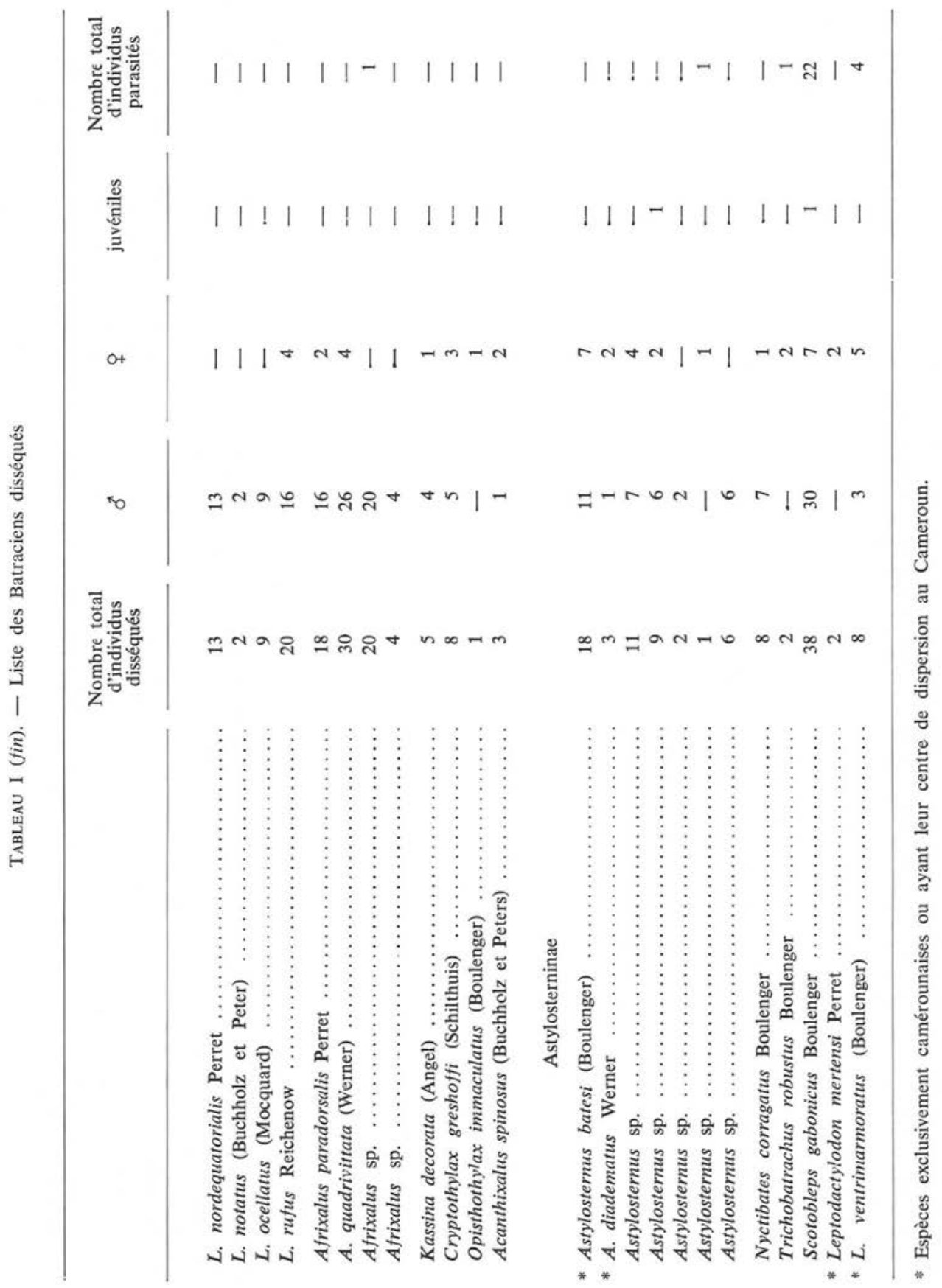


Ventouse Ventrale: $83 \mu \mathrm{m}$.

RAPPORT VO/VV: 3,6 .

APPAREIL DIGESTIF : pharynx 118-180/106-180 $\mu \mathrm{m}$; caecums intestinaux très longs. Testicules : $225-355 / 237-355 \mu \mathrm{m}$.

OVAIRE : $284-320 / 426-474 \mu \mathrm{m}$.

RÉCEPTACLE SÉMINAL : 237-225 $\mu \mathrm{m}$.

CEufs : 18,56-21,24/13,8-16,52 $\mu \mathrm{m}$ (moyenne : 19,34/15,24 $\mu \mathrm{m}$ ).

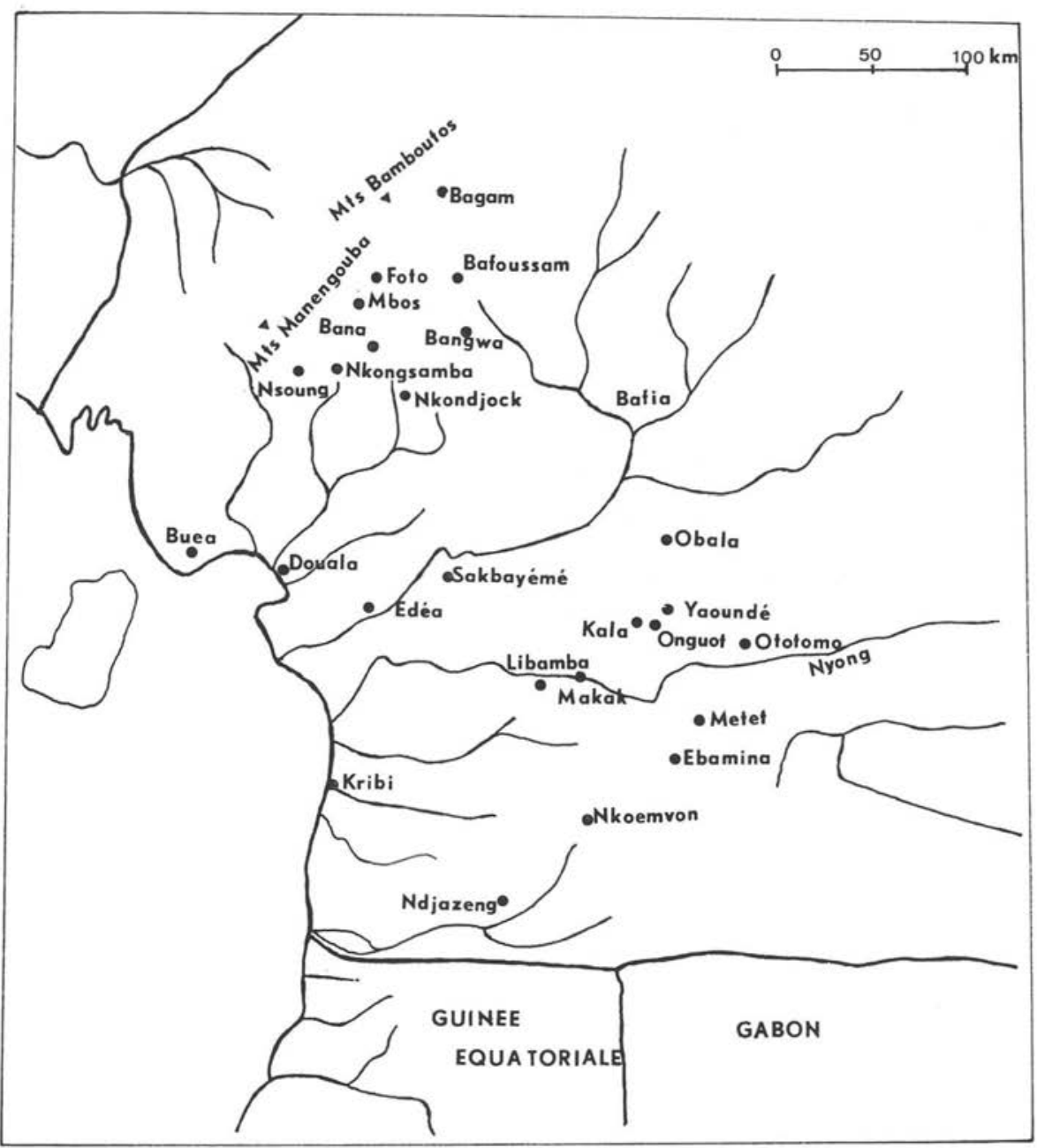

FIG. 1. - Carte indiquant les localités prospectées au Cameroun. 
Discussion: Notre matériel a les caractères typiques d'Haematoloechus darcheni Combes de Knoepffler, 1967, décrit chez Conraua crassipes au Gabon : acétabulum petit et rapport diamétral VO/VV très élevé, ovaire lobé sur son bord externe et œufs très petits.

\section{Haematoloechus micrurus}

Rees, 1964

HôTE : Dicroglossus occipitalis.

HaBITAT: poumons.

LOCALITÉ : Foulassi-Obala.

FRÉQUENCE D'INFESTATION: 1 individu parasité sur 2 disséqués.

Matériel Étudié : 2 spécimens colorés et montés in toto. Cette espèce cohabite avec Metahaematoloechus exoterorchis.

LONGUEUR: $2,2 \mathrm{~mm}$ (juv.) et $5 \mathrm{~mm}$ (adulte).

LARGEUR: 0,6 et $1,2 \mathrm{~mm}$.

CuTICULE : lisse.

Ventouse buccale: seule celle de l'individu juvénile est observable: 225/213 $\mu \mathrm{m}$.

Ventouse Ventrale : 201/194 $\mu \mathrm{m}$ (ad.) et 130/142 $\mu \mathrm{m}$ (juv.).

APPAREIL DIGESTIF : caecums intestinaux vont presque jusqu'à l'extrémité postérieure du corps.

Testicules: $193 / 387$ et $169 / 484 \mu \mathrm{m}$ (juv.), $290 / 484$ et $314 / 581 \mu \mathrm{m}$ (ad.).

OvarRe: 363/653 $\mu \mathrm{m}$ (ad.), inobservable chez le juvénile.

VITELLOGÈNES: disposés le long des caecums intestinaux.

CEuFs: 24,3-28,5/14,2-17,7 $\mu \mathrm{m}$ (moyenne 27,1/16,2 $\mu \mathrm{m}$ ).

Particularité: le corps possède un appendice postérieur.

Discussion: Bien que nous ne disposions que de deux exemplaires dont un juvénile, nous identifions cependant notre matériel à l'espèce Haematoloechus micrurus Rees, 1964, décrit chez D. occipitalis au Ghana et en Côte-d'Ivoire gràce aux caractères suivants : présence d'un appendice postérieur; utérus avec branches ascendantes et descendantes et remontant jusqu'en avant de l'ovaire.

\section{Metabaematoloechus exoterorchis}

(Rees, 1964) Yamaguti, 1971

HôTE: Dicroglossus occipitalis.

HaBitat : poumons.

Localité : Foulassi-Obala. 
FréQuenCE D'INFESTATION: 1 individu parasité sur 2 disséqués, 46 parasites Trématodes chez le même individu. Cette espèce cohabite avec Haemotoloechus micrurus.

MATÉRIEL ÉTUdié : 20 spécimens colorés et montés in toto.

LONGUEUR: $3,5-4,0 \mathrm{~mm}$.

LARGEUR : $0,9-1,2 \mathrm{~mm}$.

Cuticule: lisse.

VENTOUSE BUCCALE : 201-261/189-244 $\mu \mathrm{m}$.

Ventouse Ventrale: diam. 261-332 $\mu \mathrm{m}$.

RAPPORT VO/VV: 0,8 .

PHARYNX: 83-118/118-130 $\mu \mathrm{m}$.

APPAREIL DIGESTIF : caecums intestinaux longs.

TESTICULES : 774-1 260/194-340 $\mu \mathrm{m}$.

OVAIRE : 237-379/213-387 $\mu \mathrm{m}$.

Vitellogènes : situés le long de l'intestin de part et d'autre des testicules.

CEUFS : 23,6-27,6/15,23-18,0 $\mu \mathrm{m}$ (moyenrie : $25,85 / 16,8 \mu \mathrm{m}$ ).

PORE GÉNITAL: au niveau du pharynx.

Discussion: Notre matériel s'identifie à Metahaematoloechus exoterorchis (Rees, 1964) Yamaguti, 1971, décrit au Ghana chez D. occipitalis et en Côte-d'Ivoire. Il est caractérisé par: la ventouse ventrale plus grande que la ventouse buccale; les caecums intestinaux atteignant presque l'extrémité postérieure du corps, et surtout les testicules lobés extra-caecaux disposés en tandem.

\section{Haematoloechus ocellati}

n. sp.

HôTE: Hyperolius ocellatus purpurescens.

HaBitat : poumons.

LoCALITÉ : Nomayos.

MATÉRIEL ÉTUdié : 4 individus colorés et montés in toto.

FRÉQUENCE D’INFESTATION: 1 individu parasité sur 31 examinés.

Description: nos exemplaires présentent un corps allongé, aplati dorso-ventralement et renflé dans la moitié supérieure. Ils mesurent 5,3-6 mm de longueur sur 2-2,5 mm de largeur au niveau de la ventouse ventrale. $\mathrm{La}$ cuticule est lisse. $\mathrm{La}$ ventouse orale mesure 353 à $402 \mu \mathrm{m}$ et la ventouse ventrale, située un peu au-dessus de la moitié du corps 252-314 $\mu \mathrm{m}$. Le rapport $\mathrm{VO} / \mathrm{VV}$ est compris entre 1,2 et 1,3 . Le pharynx long de 218 à $300 \mu \mathrm{m}$ sur 274 à $314 \mu \mathrm{m}$ se poursuit par un court œsophage. Les caecums intestinaux se terminent très près de l'extrémité postérieure du corps.

Appareil reproducteur mâle: les testicules disposés en diagonale sont légèrement lobés et allongés dans le sens de l'axe du corps. Ils mesurent 1,0 à $1,7 \mathrm{~mm}$ de longueur sur 0,4 à $0,5 \mathrm{~mm}$ de largeur. La poche du cirre, difficilement observable sur nos préparations, s'étend jusqu'au bord antérieur du pharynx.

Appareil reproducteur femelle: l'ovaire volumineux et profondément lobé se trouve sur le côté gauche de la VV. Il mesure 0,8 à $1,1 \mathrm{~mm}$ de long sur 0,5 à $0,6 \mathrm{~mm}$ de large. 
Le réceptacle séminal subsphérique, situé sur le bord droit de l'ovaire, a 0,46 sur 0,58 mm. L'utérus avec ses branches ascendantes et descendantes forme des boucles extracaecales remontant jusqu'au niveau de la VV et parfois même allant en avant de celle-ci. Les vitellogènes s'étendent vers l'avant presque jusqu'à la bifurcation intestinale et vers l'arrière jusqu'à la terminaison des caecums digestifs. Les dimensions des œufs varient de 18-26 $\mu \mathrm{m}$ sur 12-15,5 $\mu \mathrm{m}$ (moyenne: 14,3-21,5 $\mu \mathrm{m}$ ).

Discussion: Par les caractères décrits ci-dessus, la morphologie de notre ver correspond à celle du genre Haematoloechus Looss, 1899.

Récemment, Batcharov et Bourgat, 1974, ont décrit une nouvelle espèce, H. combesi, chez Conraua derooi (Hulselmans, 1971) au Togo. Cette espèce s'ajoute aux trois autres espèces parasites d'Amphibiens Anoures d'Afrique, soit: $H$. micrurus Rees, 1964, chez Dicroglossus occipitalis au Ghana et en Côte-d'Ivoire ; $H$. darcheni Combes et Knoepffler, 1967, chez Conraua crassipes au Gabon, et H. lobogonadus Meskal, 1970, chez Rana angolensis en Ethiopie.

\begin{tabular}{|c|c|c|c|c|}
\hline Espèces & Rapport VO/VV & Testicules & Ovaire & Vitellogènes \\
\hline H. darcheni $\ldots \ldots$. & 4 & $\begin{array}{l}\text { faiblement lobés, } \\
0,7-08 / \\
0,48-0,6 \mu \mathrm{m}\end{array}$ & $\begin{array}{l}\text { très lobé sur lo } \\
\text { bord externe, } \\
0,46 / 0,82 \mathrm{~mm}\end{array}$ & $\begin{array}{l}\text { s'étendant en } \\
\text { arrière des testi- } \\
\text { cules. }\end{array}$ \\
\hline H. lobogonadus ..... & 2 & faiblement lobés, & lobé. & $\begin{array}{l}\text { ne s'étendant pas } \\
\text { en arrière des tes- } \\
\text { ticules. }\end{array}$ \\
\hline H. combesi $\ldots \ldots \ldots$ & 2,7 & $\begin{array}{l}\text { faiblement lobés, } \\
0,7-0,95 / \\
0,48-0,58 \mu \mathrm{m} .\end{array}$ & $\begin{array}{l}\text { lobé. } \\
0,56-0,6 \mathrm{~mm} \text {. }\end{array}$ & $\begin{array}{l}\text { s'étendant en } \\
\text { arrière des testi- } \\
\text { cules. }\end{array}$ \\
\hline H. ocellai $\ldots \ldots \ldots \ldots$ & 1,25 & $\begin{array}{l}\text { faiblement lobés, } \\
0,4-0,5 / \\
1,0-1,7 \mathrm{~mm}\end{array}$ & $\begin{array}{l}\text { profondément } \\
\text { lobé } \\
0,5-0,6 / 0,8-1,1 \mathrm{~mm}\end{array}$ & $\begin{array}{l}\text { s'étendant en } \\
\text { arrière des testi- } \\
\text { cules. }\end{array}$ \\
\hline
\end{tabular}

Nos spécimens parasites d'Hyperolius ocellatus purpurescens doivent être comparés avec $H$. darcheni, $H$. lobogonadus, $H$. combesi (voir le tableau ci-dessus). Leurs caractères discriminatifs sont: un ovaire profondément lobé et volumineux, de très grands testicules intercaecaux, un rapport ventousaire de 1,2-1,3 des vitellogènes s'étendant en arrière des testicules (fig. 2).

Par conséquent, nous considérons le parasite d'Hyperolius ocellatus purpurescens comme une espèce nouvelle pour laquelle nous proposons le nom d'Haematoloechus ocellati $\mathrm{n}$. sp. 


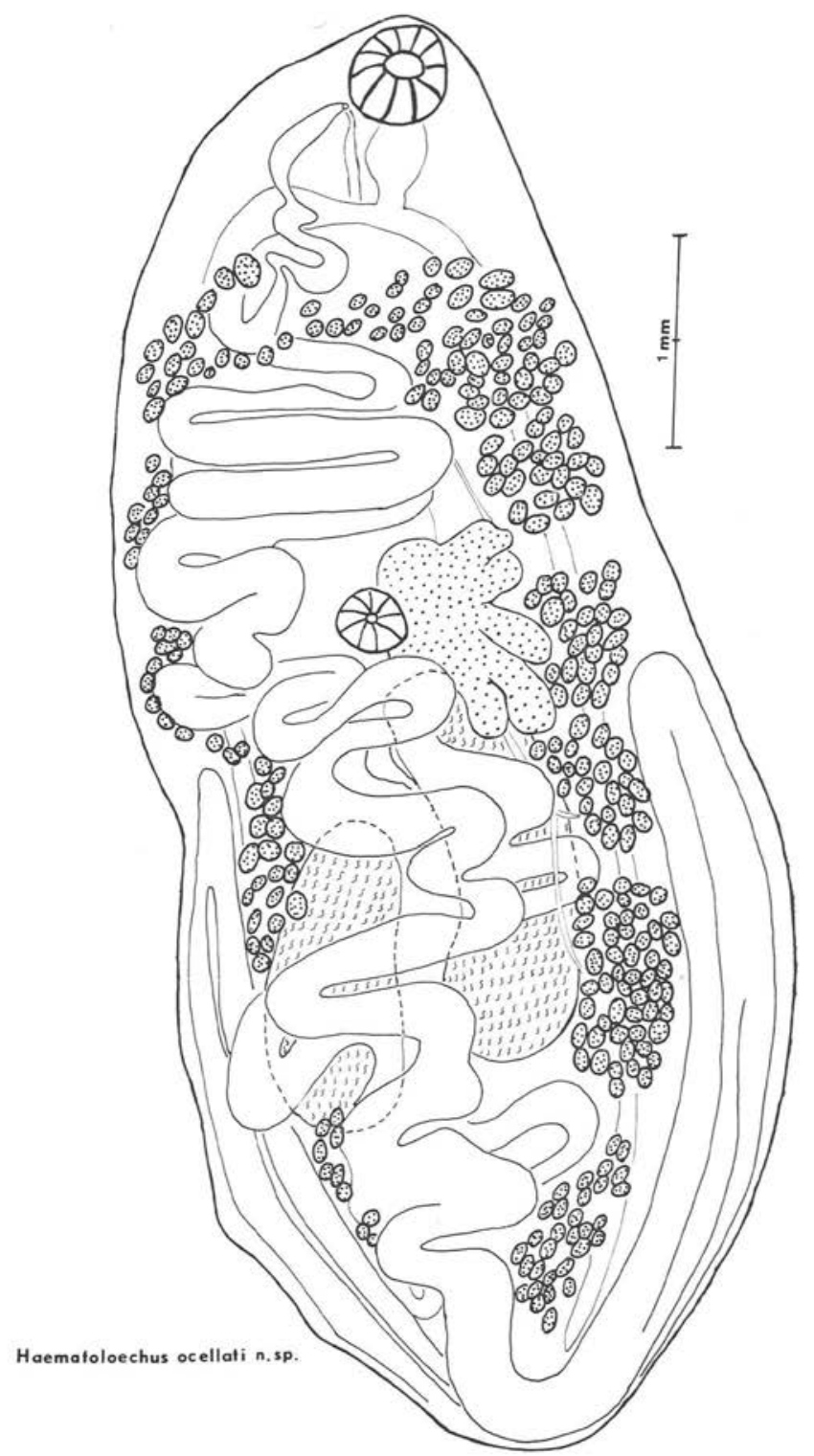

FIG. 2. - Haematoloechus ocellati n. sp. - Animal in toto, vue ventrale. 


\section{Ostioloides rappiae}

(Szidat, 1932) Odening, 1960

HôTes: Hyperolius nasutus; Hyperolius tuberculatus; Hyperolius viridistriatus; Scotobleps gabonicus.

HABITAT : intestin.

Localités: Oveng, Obala, forêt de Bakaka.

FRÉQUENCE D'INFESTATION : $8 \mathrm{H}$. nasatus parasités sur 17 disséqués; $2 \mathrm{H}$. tuberculatus parasités sur 39 disséqués; $3 \mathrm{H}$. viridistriatus parasités sur 14 disséqués; $3 \mathrm{~S}$. gabonicus parasités sur 38 disséqués.

MATÉRIEL ÉTUdié : 10 spécimens colorés et montés in toto.

LONGUEUR: $2,4-4,3 \mathrm{~mm}$.

LARGEUR: $1,3-1,8 \mathrm{~mm}$.

VENTOUSE ORALE : $218-290 \mu \mathrm{m} / 218-266 \mu \mathrm{m}$.

VeNTOUSE VENTRALE : $266-339 \mu \mathrm{m} / 195-339 \mu \mathrm{m}$.

RAPPORT VO/VV: 0,8-0,9.

Cuticule: spinulée jusqu'à la moitié postérieure du corps.

APPAREIL DIGESTIF : les caecums intestinaux atteignent les $3 / 4$ du corps.

Appareil Génital $\delta^{*}$ : testicules: 242-314 $\mu \mathrm{m} / 213-319 \mu \mathrm{m}$. Poche du cirre: 415 $426 \mu \mathrm{m}$ de long.

APPAREIL GÉNITAL $q$ : ovaire : $242-363 \mu \mathrm{m} / 218-339 \mu \mathrm{m}$. Vitellogènes : $35-64 / 24-43 \mu \mathrm{m}$. Les glandes vitellogènes s'étendent jusqu'à l'extrémité postérieure des caecums. CEufs : 27,14-29,15/19,98-16,52 $\mu \mathrm{m}$ (moyenne : 30,14/15,7 $\mu \mathrm{m}$ ).

Discussion: Notre Digène répond à la description d'Ostioloides rappiae (Szidat, 1932) Odening, 1960, trouvé au Liberia chez Rappia concolor, notamment par les caractères suivants: ventouse ventrale plus grande que la ventouse orale, testicules disposés l'un à côté de l'autre vers le milieu du corps, follicules vitellogènes formant deux champs s'étendant de la bifurcation intestinale jusqu'à la terminaison des caecums.

$H$. tuberculatus, $H$. nasutus, $H$. viridistriatus et $S$. gabonicus sont des hôtes nouveaux pour ce parasite.

\section{Plagitura eburnense}

(Maeder, 1969) n. comb.

HôTES ET FRÉQUENCE D'INFESTATION: Bufo latifrons: 1 parasité sur 9 disséqués; Hylarana lepus: 1 parasité sur 24 disséqués; Hylarana longipes: 1 parasité sur 4 disséqués; Hylarana sp.: 3 parasités sur 10 disséqués; Hylarana sp.: 3 parasités sur 11 disséqués; Cardioglossa gracilis: 1 parasité sur 5 disséqués; Phrynobatrachus auritus: 1 parasité sur 4 disséqués; Ph. batesi : 1 parasité sur 1 disséqué ; Ph. steindachneri : 3 parasités sur 13 disséqués; Pedropedetes cameronensis: 1 parasité sur 3 disséqués; Astylosternus batesi : 2 parasités sur 18 disséqués; Astylosternus sp.: 1 parasité sur 1 disséqué; Trichobatrachus robustus: 1 parasité sur 2 disséqués; Leptodactylodon ventrimarmoratus: 4 parasités sur 8 disséqués. 
InfEstations doubles: P. eburnense et M. monas chez Hylarana sp., B. latifrons et $A$. batesi.

Habitat: intestin.

Localıtés: Bagam, Bangwa, Bambutos, Forêt de Bakaka, Kala, Maholé, Ndjazeng, Ndoungué, Nkoemvon, Nkongsamba, Nkondjock, Nsoung, Sakbayémé.

MATÉRIEl ÉtUdí́ : 25 exemplaires colorés et montés in toto.

LONGUEUR: $0,9-1,5 \mathrm{~mm}$.

LARGEUR: $0,5-0,9 \mathrm{~mm}$.

Cuticule : épineuse.

VENTOUSE BUCCALE : 142-237/130-189 $\mu \mathrm{m}$.

Ventouse Ventrale: 142-201/130-189 $\mu \mathrm{m}$.

RAPPORT VO/VV: $1,0-1,1$.

APPAREIL DIGESTIF : caecums intestinaux atteignent les $2 / 3$ du corps.

TESTICULES : $95-178 / 73-178 \mu \mathrm{m}$.

OVAIRE: $118-237 / 102-189 \mu \mathrm{m}$.

Vitellogènes: répartis sur tout le corps, du pharynx à l'extrémité postérieure.

EUFS : 40,1-51/23,6-29,5 $\mu \mathrm{m}$ (moyenne : 43,3/26,3 $\mu \mathrm{m}$ ).

Discussion: Notre matériel présente les caractères discriminatifs et les mensurations de Haplometroides eburnense Maeder, 1969, soit: la position constante des testicules en arrière des caecums, l'extension des vitellogènes jusqu'à l'extrémité postérieure du corps, la position de la poche du cirre sur le côté de la ventouse ventrale. Cette espèce a été retrouvée au Gabon par Maeder, Combes et Knoepffiler, $1970 \mathrm{~b}$. Tous les hôtes en provenance du Cameroun sont des hôtes nouveaux pour cette espèce.

Dans sa diagnose du genre Haplometroides, dont l'unique espèce $H$. bucciocola est parasite de la cavité buccale d'un serpent d'Amérique du Sud, Odhner (1911) note que les testicules sont disposés l'un derrière l'autre et intercaecaux. L'attribution de H. eburnense Maeder, 1969, ne nous paraît pas judicieuse, parce que les testicules ne sont ni intercaecaux, ni disposés l'un derrière l'autre, chez le parasite africain. Au contraire, comme ceux de Maeder (1969), nos spécimens offrent de grandes ressemblances avec le genre Plagitura Holl, 1928, parasite de l'intestin d'Amphibiens Urodèles américains et qui présente la même disposition des testicules. Il nous paraît logique, dans l'état actuel de nos connaissances de ce groupe, de proposer le transfert de $H$. eburnense dans le genre Plagitura. La diagnose de ce genre doit être quelque peu modifiée quant à l'extension des vitellogènes qui occupent un champ beaucoup plus vaste chez le parasite africain que chez Plagitura intermedia Etges et Bonner, 1965, P. parva Stunkard, 1933, et $P$. salamandra Holl, 1928, où ils sont disposés latéralement le long des caecums. Yamaguti, 1971, considère Plagitura Holl, 1928, syn. de Manodistomum Stafford, 1905. Cette opinion ne nous paraît pas logique puisque la diagnose de Manodistomum se fonde sur la description incomplète d'un spécimen immature, comme le relève Stunkard (1932).

$P$. eburnense est de toute manière différent des autres espèces du genre par l'étendue des vitellogènes. 


\section{Mesocoelium gabonensis}

Maeder, Combes et Knoepffler, 1969

HôTES: Arthroleptis poecilonotus; A. variabilis; Arthroleptis sp.; Astylosternus batesi. HABIтAT: intestin.

Localités: Nsoung, Ndjazeng, Kala, Bafia.

FRÉQUENCE D'INFESTATION: $1 \mathrm{~A}$. poecilonotus parasité sur 3 disséqués; $1 \mathrm{~A}$. variabilis parasité sur 5 disséqués; $1 \mathrm{~A}$. $s p$. parasité sur 3 disséqués; $2 \mathrm{~A}$. batesi parasités sur 18 disséqués.

Matériel Étudié : 14 spécimens adultes et 1 juv. colorés et montés in toto.

LONGUEUR : $0,9-2,2 \mathrm{~mm}$.

LARGEUR: $0,5-1,0 \mathrm{~mm}$.

Cuticule: spinulée dans le tiers antérieur.

VENTOUSE BUCCALE: 272-458/249-435 $\mu \mathrm{m}$.

Ventouse VenTRALE: 106-249/112-249 $\mu \mathrm{m}$.

RAPPORT VO/VV: $1,5-2,1$.

TESTICULES : $83-190 / 76-166 \mu \mathrm{m}$.

OVAIRE: $102-213 / 90-225 \mu \mathrm{m}$.

EUfS : $35,4-47,0 / 17,7-27,0 \mu \mathrm{m}$ (moyenne : 38,3/23,8 $\mu \mathrm{m}$ ).

Discussion: Les caractères principaux de l'espèce: longs caecums intestinaux se prolongeant très au-delà des vitellogènes, rapport $\mathrm{VO} / \mathrm{VV}$ compris entre 1,5 et 2,1 , grands œufs, se retrouvent constamment chez les parasites examinés. Nous l'identifions donc à $M$. gabonensis. Ce parasite n'avait pas encore été signalé chez $A$. poecilonotus, A. variabilis, $A$. arthroleptis sp. et $A$. batesi.

\section{Mesocoelium monas}

Freitas, 1958

Hôtes ET FRÉQUENCE D'INFESTATION: Bufo maculatus: 11 parasités sur 28 disséqués; B. regularis: 6 parasités sur 14 disséqués; $B$. latifrons: 2 parasités sur 9 disséqués; Hylarana albolabris: 5 parasités sur 24 disséqués; $H$. lepus: 1 parasité sur 24 disséqués; Hylarana $s p .: 4$ parasités sur 13 disséqués; Hylarana $s p$.: 5 parasités sur 11 disséqués ; Ptychadena mascareniensis : 26 parasités sur 31 disséqués ; Pt. oxyrhynchus : 5 parasités sur 9 disséqués; Pt. perreti: 2 parasités sur 3 disséqués; Pedropedetes johnstoni: 1 parasité sur 16 disséqués; Astylosternus diadematus: 1 parasité sur 3 disséqués; A. batesi : 3 parasités sur 18 disséqués; Scotobleps gabonicus: 19 parasités sur 38 disséqués.

INFESTATIONS DOUbLes: $M$. monas et $H$. eburnense chez Hylarana sp.; Bufo latifrons et $A$. batesi.

HaBITAT : intestin.

Localıtés: Bakaka, Bangwa, Buea, Falaise des Mbos, Kala, Libamba, Maholé, Ndabock, Ndjazeng, Ndoungué, Nkoemvon, Nkongsamba, Ototomo, Rte d'Edea, Sakbayémé, Yaoundé. 
Matériel ÉTUdié : 40 spécimens colorés et montés in toto.

LONGUEUR : $1,2-3,3 \mathrm{~mm}$.

LARGEUR: $0,4-1,2 \mathrm{~mm}$.

Cuticule: spinulée.

Ventouse BUCCALE : 190-450/200-414 $\mu \mathrm{m}$.

Ventouse Ventrale: 123-296/137-272 $\mu \mathrm{m}$.

RAPPORT VO/VV: 1,1-1,6.

Testicules : $83-225 / 88-237 \mu \mathrm{m}$.

OVAIRE: $95-237 / 95-237 \mu \mathrm{m}$.

CEuFs: 29,5-44,8/16,5-25,9 $\mu \mathrm{m}$ (moyenne : 37,2/21,9 $\mu \mathrm{m}$ ).

Discussion: Ces Digènes infestant des hôtes variés présentent tous les caractères spécifiques de Mesocoelium monas Freitas, 1958, soit: rapport ventousaire VO/VV nettement inférieur à 2, circonvolutions utérines atteignant presque l'extrémité postérieure du corps, caecums courts.

B. latifrons, A. diadematus, A. batesi, S. gabonicus et Hylarana sp. sont des hôtes nouveaux.

\section{Diplodiscus subclavatus}

(Goeze, 1782)

HôTES: Dicroglossus occipitalis; Ptychadena mascareniensis; Leptopelis aubryi.

HABITAT: rectum.

LOCALITÉ : Foulassi-Obala.

FRÉQUENCE D'INFESTATION: 2 D. occipitalis parasités sur 2 disséqués; D. subclavatus cohabitait avec Haematoloechus micrurus; 1 Pt. mascareniensis parasité sur 31 disséqués; 1 L. aubryi parasité sur 9 disséqués.

Matériel Étudié : 3 individus colorés et montés in toto.

Description :

LONGUEUR : $1,2-2,7 \mathrm{~mm}$.

LARGEUR: $0,9-1,6 \mathrm{~mm}$.

CuTICULE: lisse.

Ventouse BuCCALE: diamètre 166-266 $\mu \mathrm{m}$.

DISQUE POSTÉRIEUR: diamètre 920-1 $137 \mu \mathrm{m}$.

VeNTOUSE POSTÉRIEURE: chez 1 individu, diamètre $218 \mu \mathrm{m}$.

DiverTicules PHARYNGIENS: longueur 427-462 $\mu \mathrm{m}$.

APPAREIL DIGESTIF : caecums courts.

OVAIrE: mesurable sur 1 seul spécimen, 193/266 $\mu \mathrm{m}$.

Testicule: mesurable sur 1 seul individu, 460/290 $\mu \mathrm{m}$.

EUfs: $118,5-137,5: 76-99,5 \mu \mathrm{m}$ (moyenne : 125,35/83,66 $\mu \mathrm{m}$ ).

Discussion: Nous identifions nos spécimens à l'espèce Diplodiscus subclavatus (Goeze, 1782) par: ses caecums longs, son pore génital au niveau ou en avant de la bifurcation intestinale, ses vitellogènes s'étendant jusqu'au bulbe œsophagien. 
L'espèce D. subclavatus a déjà été signalée par Skrjabin (1916) chez Bufo sp. en Afrique centrale, par Joyeux et Baer (1928) chez Bufo regularis au Dahomey, par Balozet et Callot (1938) chez Rana ridibunda en Tunisie, par Maeder et al. (1969) chez Phlyctimantis leonardi au Gabon et par Maeder (1973) chez Dicroglossus occipitalis en Côte-d'Ivoire. Pt. mascareniensis et L. aubryi sont des hôtes nouveaux.

\section{Progonimodiscus doyeri}

(Ortlepp, 1926)

HôTE: Conraua crassipes.

HABITAT: rectum.

LOCALITÉ: Ebamina.

FréQuenCE D'INFESTATION: 1 individu parasité sur 10 disséqués. Le même hôte hébergeait également un parasite pulmonaire Haematoloechus darcheni.

Matériel Étudié : 2 spécimens colorés et montés in toto.

Description :

LONGUEUR : $1,5 \mathrm{~mm}$.

LARGEUR: $0,5-0,6 \mathrm{~mm}$ au milieu du corps.

Ventouse BUCCALE: $142 \mu \mathrm{m}$ de diamètre.

DIVERTICULES PHARYNGIENS: $190 \mu \mathrm{m}$ de long sur $178 \mu \mathrm{m}$ de large.

ESOPHAGE : 296-308 $\mu \mathrm{m}$ de long.

APPAREIL DIGESTIF : caecums courts.

Disque postérieur: $0,8-1 \mathrm{~mm}$ de diamètre. Il renferme une ventouse dont le pourtour est découpé.

Testicules et ovaires : ne sont pas mesurables.

EUfF : $80,2-94,2 / 37,7-42,4 \mu \mathrm{m}$ (moyenne : 87,9/44,7 $\mu \mathrm{m}$ ).

Discussion: Notre matériel ne se prête pas à une description plus détaillée. Nous l'identifions à Progonimodiscus doyeri (Ortlepp, 1926), parasite de Xenopus laevis laevis en Afrique du Sud, retrouvé chez le même hôte par Beverley-Burton (1962) en Rhodésie. Cette espèce est caractérisée comme nos spécimens par des caeca très courts, des vitellogènes postcaecaux et la ventouse postérieure très découpée. Conraua crassipes est un hôte nouveau pour cette espèce.

\section{Conclusion}

Les Helminthes des Amphibiens Anoures de l'Afrique équatoriale sont actuellement assez bien connus. Ainsi, ces dernières années, de nombreuses contributions ont été apportées à l'étude des Trématodes de la Côte-d'Ivoire, du Gabon, du Ghana, 
de la République centrafricaine et du Togo. Concernant le Cameroun, nous n'avons trouvé que les travaux de Dollfus (1929) et Saoud (1964).

Par sa situation géographique, ce pays constitue une zone intermédiaire entre l'Afrique occidentale et orientale; sa faune batrachologique est riche et variée. Elle comprend de nombreuses espèces endémiques ; cependant, beaucoup ont une aire de distribution qui s'étend aux pays voisins: Guinée équatoriale, Congo, Gabon, Nigeria, etc. Sur le nombre total d'Amphibiens examinés (environ 800), 244 individus appartenant à 37 espèces différentes hébergeaient des Trématodes. Nous avons retrouvé neuf parasites déjà signalés sur le continent africain. Nous décrivons une espèce nouvelle, $H$. ocellati n. sp., et proposons le transfert d'Haplometroides eburnense Maeder, 1969, dans le genre Plagitura Holl, 1928.

La biogéographie des hôtes et des parasites, de même que leur distribution chez les différents Amphibiens, permettent de tirer les conclusions suivantes:

- Chez les Haematoloechinae, la spécificité parasitaire est très stricte ; H. micrurus et Metahaematoloechus exoterorchis parasitent toujours le même hôte, D. occipitalis; la distribution du parasite semble être identique à celle de l'hôte, qui vit dans les zones limites forêt-savane du Sénégal à l'Ethiopie. Si la distribution de $H$. darcheni semble beaucoup plus limitée, c'est que son hôte, Conraua crassipes, n'existe que dans la zone forestière méridionale du Cameroun, du Gabon et du Congo. Ici aussi, la répartition de l'hôte et du parasite est la même.

- Ostioloides rappiae, parasite surtout les Hyperoliinae du Liberia au Cameroun; ses hôtes de prédilection sont des Amphibiens savanicoles ou vivant au contact de la forêt. Nous signalons pour la première fois ce parasite chez un Astylosterninae, $S$. gabonicus, qui est fréquent dans toute la zone forestière du Nigeria au Congo.

- $P$. eburnense a une distribution très comparable à l'espèce précédente puisqu'on la rencontre de la Côte-d'Ivoire à la République centrafricaine. Mais elle est peu spécifique, parasitant des Bufonidae et des Ranidae (Raninae, Arthroleptinae, Phrynobatrachinae, Astylosterninae, Hyperoliinae). Beaucoup de ces hôtes ont en commun le fait de vivre en zone forestière et au bord des eaux courantes. Nous signalons $P$. eburnense chez 14 hôtes nouveaux.

Les Mesocoeliidae sont représentés au Cameroun par deux espèces: la première, M. gabonensis, signalée jusqu'ici uniquement au Gabon et en République centrafricaine chez plusieurs Ptychadena, fréquents en Afrique équatoriale. Ce parasite semble être localisé à l'Afrique centrale. Nous l'avons trouvé d'autre part chez des hôtes nouveaux du genre Arthroleptis, petits Anoures vivant dans les formations secondaires, défrichements et plantations, ou dans les forêts primaires de basse montagne, et chez Astylosternus batesi, espèce camerounaise du Sud forestier. La deuxième espèce, $M$. monas, a une aire de distribution remarquablement vaste, tropicale et subtropicale. Elle parasite près de 30 espèces différentes d'Anoures, très diverses du point de vue écologique. Cet Helminthe, déjà signalé au Cameroun chez $P$. mascareniensis par Saoud (1964) sous le nom de $M$. monodi Dollfus, 1929, a été retrouvé chez 14 hôtes différents dont cinq sont nouveaux, ayant leur centre de dispersion au Cameroun. 
Parmi les parasites du rectum, Diplodiscus subclavatus est signalé en Afrique centrale, au Dahomey, en Côte-d'Ivoire, au Gabon et en Tunisie. Nous le retrouvons au Cameroun chez deux hôtes nouveaux : P. mascareniensis et L. aubryi. Progonimodiscus doyeri a une aire de distribution très différente: Madagascar, Afrique du Sud, Rhodésie et Cameroun. Il s'agit de la seule espèce parmi celles que nous avons rencontrées qui vive dans la moitié sud du continent africain.

\section{REMERCIEMENTS}

Au terme de ce travail, nous exprimons une pensée reconnaissante à la mémoire du $\mathrm{P}^{\mathrm{r}}$ J.-G. Baer, qui nous a encouragé à faire ce séjour au Cameroun pour y récolter du matériel.

Nos vifs remerciements s'adressent au $\mathrm{P}^{r}$ C. Vaucher pour ses conseils.

\section{Bibliographie}

Batcharov (G.) et Bourgat (R.), 1974. - Haematoloechus combesi n. sp. (Trematoda, Haematoloechidae), parasites d'Amphibiens Anoures au Togo. Ann. Parasit. hum. comp., 49, 337-342.

Combes (C.) et KnoepfFler (L.-P.), 1967. - Parasites d'Amphibiens du Gabon Haematoloechidae (Digenea) Biologica Gabonica, 3, 141-147.

Dor lfus (R.-Ph.), 1929. - Contribution à l'étude de la faune du Cameroun par Th. Monod (fasc. 2). Helmintha I. Trematoda et Acanthocephala. Faune Colon. fr., 3, 73-114.

ETGes (F. J.) et Bonner (T. P.), 1965. - Plagitura intermedia sp.n. (Trematoda: Plagiorchiidae) from the common newt, Notophthalmus viridescens viridescens. J. Parasit., $51,775-776$.

Fischthal (J.-H.) et Thom $/$ s (J.-D.), 1968. - Digenetic Trematodes of Amphibiens and Reptiles from Ghana. Proc. helminth. Soc. Wash., 35, 1-15.

Maeder (A.-M.), 1969. - Trématodes de Batraciens de Côte d'Ivoire. Revue suisse Zool., 76, 903-918.

MAEder (A.-M.), 1973. - Monogènes et Trématodes parasites d'Amphibiens en Côte d'Ivoire. Revue suisse Zool., 80, 268-322.

Maeder (A.-M.), Combes (C.) et Knoepffler (L.-P.), 1969 a. - Parasites d'Amphibiens du Gabon: Mesocoeliidae (Digenea). Taxonomie et chorologie du genre Mesocoelium Odhner, 1911. Biologica Gabonica, 5, 289-303. - 1969 b. — Parasites d'Amphibiens du Gabon: Plagiorchiidae et Paramphistomatidae (Digenea). Biologica Gabonica, 5, 283-288.

Mafder (A.-M.), $1970 a$. - Parasites d'Amphibiens de Côte d'Ivoire: Plagiorchiidae et Mesocoeliidae (Digenea). Biologica Gabonica, 4, 387-393. - 1970 b. - Parasites 
d'Amphibiens de République Centrafricaine: Plagiorchiidae et Mesocoeliidae (Digenea). Biologica Gabonica, 4, 394-402.

Odening (K.), 1960. - Trematoda, Digenea. Plagiorchiidae III (Haematoloechinae) und Omphalometridae. Tierreich, 75, 77.

Odhner (Th.), 1911. - Nordostafrikanische Trematoden. Res. Swed. Zool. Exp. to Egypt and White Nile, 1901, 4, 1-165.

ReEs (G.), 1964. - Two new species of the genus Haematoloechus (Digenea: Plagiorchiidae) from Rana occipitalis (Günther) in Ghana. Parasitology, 54, 345-368.

SAoud (M. F.), 1964. - On some Digenetic Trematodes (Mescoelium spp.) from Amphibia and Reptilia of the Cameroons. J. Helminth., 38, 291-302.

Stafford (J.), 1905. - Trematodes from Canadian Vertebrates. Zool. Anzeig., 28, 681694.

Stunkard (H. W.), 1936. - The Morphology and Life History of Plagitura parva Stunkard, 1933 (Trematoda). J. Parasitol., 22, 354-374.

Vercammen-Grandjean (P.-H.), 1960. - Les Trématodes du Lac Kivu Sud (Vermes). Ann. Mus. Roy. Afr. Centrale, Tervuren. $N^{\text {ue }}$ série, in-4 ${ }^{\circ}$, Sc. Zool., 5, 1-171.

Yamaguti (S.), 1971. - Synopsis of Digenetic Trematodes of Vertebrates. Keigaku Publ. Tokyo, 1074 p. 